\title{
Diseño e implementación de un entrenador en lista de instrucciones para la programación de PLCs
}

\author{
John W. Vásquez C. \\ Eliana Torres N. \\ Johnnatan Castañeda
}

Recibido el 10 de febrero de 2009. Aprobado el 15 de abril de 2009

\begin{abstract}
Resumen
\end{abstract}
El presente proyecto, radica en el diseño, construcción y puesta en servicio de un entrenador de PLC en lenguaje AWL o lista de instrucciones, conformado por 3 tarjetas, cada una con 8 entradas y 8 salidas digitales a relevador, basado en un microcontrolador de gama media PIC $18 F 4550$ que permite la comunicación con un PC vía puerto USB. El entrenador en lista de instrucciones cuenta con software de programación y una interfase grafica con el usuario

\section{Palabras clave}

PLC, Lista de instrucciones, AWL, Microcontrolador, USB, Software

Abstract

This project consists of the design, implementation and putting of service of a trainer of PLC of language AWL or instruction list, that coast of 3 cards of 8 income and 8 digital exits to relay. Based on a microcontroller of average range PIC $18 F 4550$ that allows the communication with PC route port USB. The trainer in list of instructions possesses (relies on) software of programming and a graphical interface.

\section{Key words}

PLC, Intructions list, AWL, Microcontroller, USB port, Software 


\section{Introducción}

Dentro de las actuales necesidades de la Facultad de Ingeniería de Uniminuto, se encuentra el mejoramiento y fortalecimiento de los laboratorios, en especial en aquellos donde se imparten asignaturas relacionadas con el control de procesos y controladores lógicos programables PLCs. Este trabajo propone el diseño, desarrollo e implementación de un software simulador entrenador para PLC's (Controladores Lógicos Programables) en AWL (Lista de instrucciones), incluyendo tres tarjetas de entradas y salidas digitales, así como el hardware necesario para el acondicionamiento de las señales de entrada y salida.

En la mayoría de los casos a escala industrial la falta de conocimiento por parte de los técnicos o tecnólogos en los lenguajes de programación de los autómatas programables o PLCs dificulta su desempeño satisfactorio en la compañía, obligando a las mismas a recurrir a personal experimentado y con algún conocimiento sobre el tema; dejando por fuera al técnico que no posee estos conocimientos.

Con la implementación de los entrenadores en lista de instrucciones, los estudiantes tendrán la posibilidad de manipular tecnologías enfocadas a este campo de la programación y el control adquiriendo competencias que le serán de gran utilidad al momento de enfrentarse al mundo laboral dado que cada día es mas común ver la aplicación de los PLCs, no solo en la industria sino también en la vida cotidiana, por ejemplo en los edificios inteligentes y los nuevos dispositivos diseñados para el hogar que cuentan con controles cada vez más sofisticados.

Los entrenadores dan la oportunidad al estudiante de familiarizarse con equipos de control industrial, así como de crear incentivos para la investigación en los temas de automatización industrial.

\section{Metodología}

Para el desarrollo del proyecto se analizaron productos existentes en el mercado industrial que orientan su funcionamiento a la implementación y utilización de microcontroladores como parte fundamental de su arquitectura. Como elemento básico se utilizó el PIC $18 \Varangle 4550$ por sus características tanto eléctricas, funcionales y de programación.

El diseño del entrenador se inicia conociendo y descartando los componentes necesarios para la comunicación del microcontrolador con el exterior y definiendo las etapas que constituyen el sistema, dividiéndolas y clasificándolas por bloques.

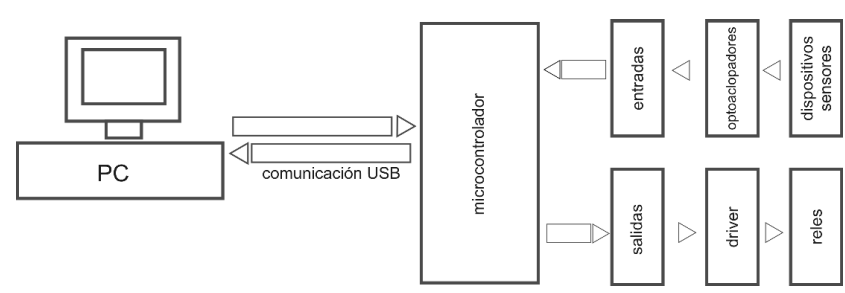

Figura 1. Diagrama a bloques del entrenador. Fuente: Los autores

\section{Diseño}

El entrenador en lista de instrucciones o AWL se diseña de forma tal que el usuario pueda familiarizarse con la programación de los PLCs reales, con la diferencia de que las conexiones son más sencillas y reducidas.

El diseño de las tarjetas se realizó a través de un software de simulación llamado PROTEUS, el cual brinda la posibilidad de desarrollar el circuito electrónico, simular su funcionamiento, diseñar el circuito impreso y poder visualizar en 3D el producto final.

Para cada etapa del sistema se diseña un circuito y se construye una tarjeta, dando la posibilidad de reducir la robustez del circuito y permitiendo que sea mas compacto y estético, además de permitir la detección y corrección de posibles fallas en el funcionamiento de ésta.

Las entradas digitales de la tarjeta es a $24 \mathrm{Vdc}$ que es un estándar a nivel industrial, de esta manera los estudiantes pueden conectar los sensores que se encuentran en el laboratorio de control.

Las señales son acondicionadas de tal manera que el microcontrolador reciba señales de tipo TTL (Transitor Transistor Logic) de 0 a 5 Vdc; esto se hace a través de una etapa de opto acoplado previamente calculado. El micro controlador se encarga de recibir la información del programa establecido por el estudiante, enviar el estado de las entradas y salidas y de activar o desactivar las salidas correspondientes a la programación establecida en la lista de instrucciones, la salida a relé permite la utilización de diferentes valores de voltajes para los actuadores.

Las salidas de la tarjeta serán a relevador. Para este proceso, es necesario volver a realizar un acondicionamiento de las señales, usando un dispositivo de potencia para la activación de los relés. Con la utilización del ULN 2803 no hay problema puesto que 
este dispositivo puede manejar corrientes más altas que el PIC (micro controlador).

\section{Desarrollo \\ Etapa de opto acoplado y microprocesado}

En la implementación del PLC, se deben usar los protocolos o estándares a nivel industrial. El primero de ellos son los niveles de voltaje utilizados, en los PIC's por ser tecnología TTL el nivel es de 5 VDC, mientras que en la industria son de 24 VDC lo que hace necesario un acondicionamiento de las señales. Para esto se utiliza un opto acoplador en las entradas del micro que permite relevar de 24 a 5 VDC. El opto utilizado es 4N25 por sus características eléctricas.
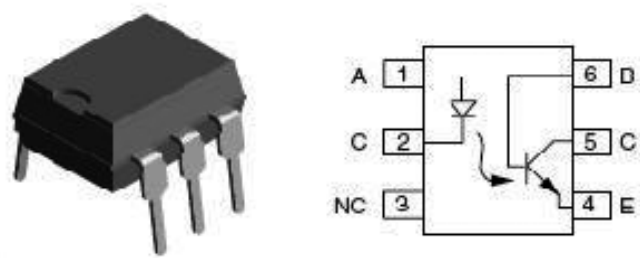

Figura 2. Opto aclopador 4N25. Fuente: National Semiconductors

El funcionamiento del opto acoplador es similar al transistor NPN y cuentan con base, emisor y colector. En la base tiene un LED infrarrojo lo cual pone un límite a la corriente y el voltaje que se le puede aplicar en la base.

La conexión de una resistencia de 10k en el ánodo del Led de la base del opto acoplador permite un divisor de voltaje en su entrada con los $24 \mathrm{Vdc}$ de señal de entrada, de esta forma el voltaje que cae en el Led de la base será al alrededor de $5 \mathrm{Vdc}$ y la corriente que circula a través de él será de 1,9 mA.

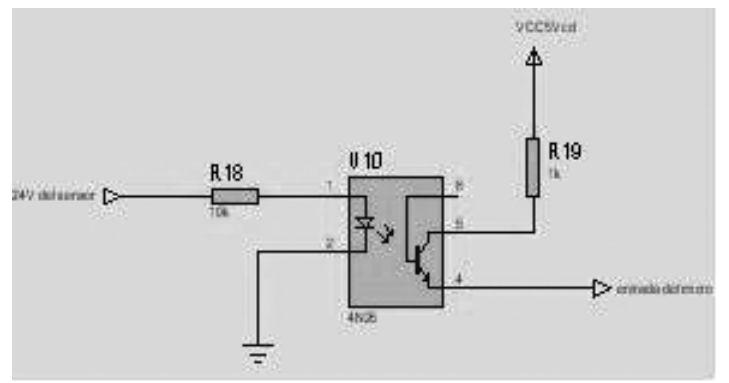

Figura 3. Diagrama de la conexión de una resistencia de $10 \mathrm{~K}$ al opto acoplador. Fuente: Los autores

El opto funciona como un transistor en corte y saturación, haciendo las veces de interruptor que envía $5 \mathrm{~V}$ tomados del puerto USB hacia la entrada correspondiente del micro controlador. Se limita la corriente de colector con una resistencia de $1 \mathrm{~K}$ aprox. Conectando un led en paralelo para visualizar el correcto funcionamiento de esta etapa.

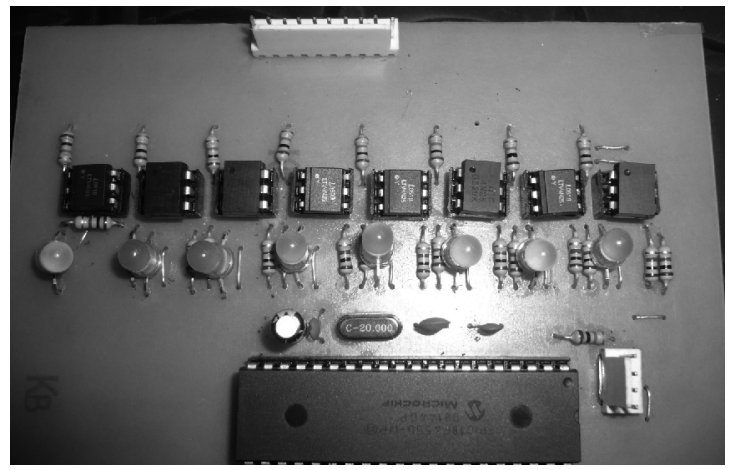

Figura 4. Relés opto acoplados en el prototipo real. Fuente: Los autores

\section{Conexión de micro controlador}

El micro controlador se alimenta por medio del puerto USB, ya que este suministra 5 y 0 voltios VDC; lo que ahorra la utilización de una fuente externa. La configuración convencional del PIC 18F4550 es similar a la utilizada en el PIC 16F877A.

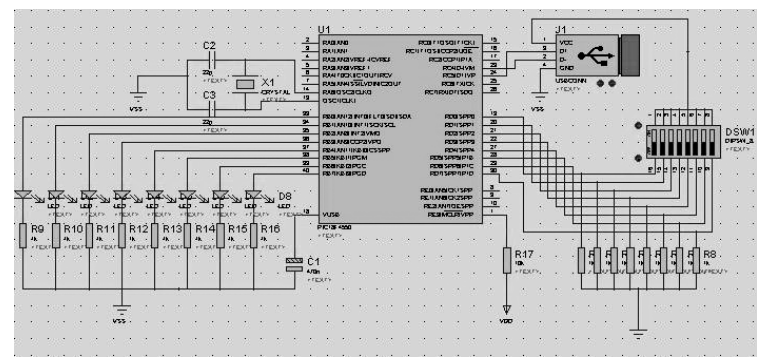

Figura 5. Diagrama de configuración del micro controlador en el prototipo del entrenador. Fuente: Los autores

El PIC 18F4550, además de la configuración para su funcionamiento, requiere de componentes adicionales para la comunicación por puerto USB con un computador. Estos consisten en un condensador electrolítico en el pin Vusb, así como de las señales D+ y D- del puerto USB.

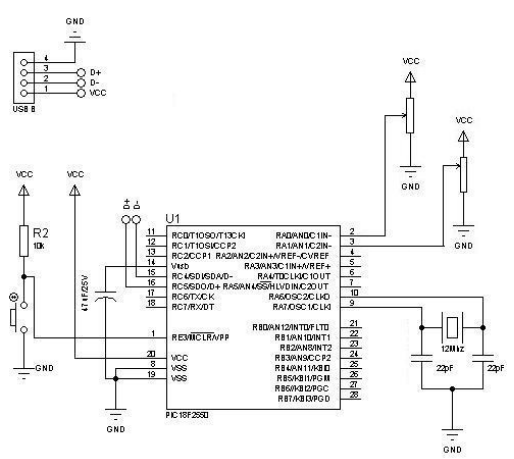

Figura 6. Diagrama de conexión del puerto USB al PIC. Tomado de http://micros.mforos.com/1149901/6229274-problema-con-comunicacion-pc-pic-por-usb/ 


\begin{tabular}{|c|c||c||c|}
\hline PIN & NOMBRE & COLOR DEL CABLE & DESCRIPCIÓN \\
\hline \hline 1 & VCC & ROJO & + V5 \\
\hline 2 & $D-$ & BLANCO & Data- \\
\hline 3 & $D+$ & VERDE & Data+ \\
\hline 4 & GND & NEGRO & Tierra \\
\hline
\end{tabular}

Cuadro 1. Descripción del cable USB

Tomado de http://es.wikipedia.org/wiki/Bus_de_Serie_Universal

Una vez realizadas las pruebas de comunicación USB, se continúo con la etapa en la cual se define la metodología y el modelo de desarrollo, analizando el sistema actual y cada proceso.

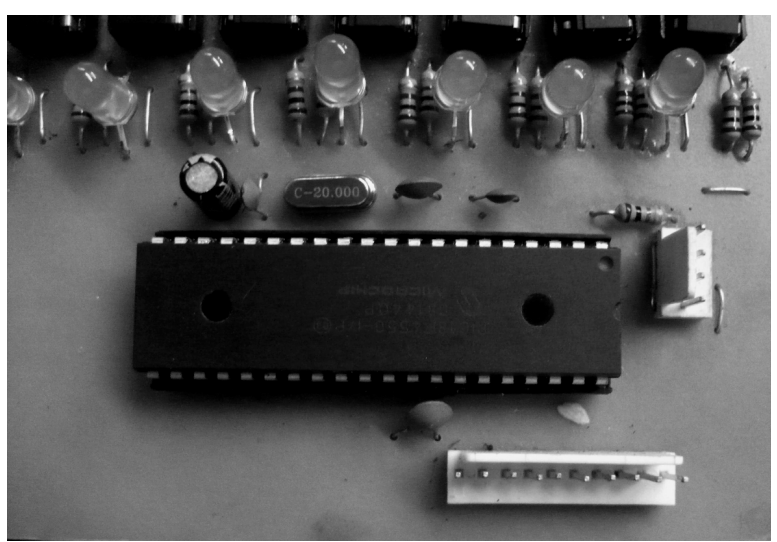

Figura 7. Micro controlador en el prototipo del entrenador. Fuente: Los autores

La primera fracción de la tarjeta es la parte de control la cual contiene una sección de opto acoplado de las señales y el micro controlador con la conexión USB hacia el PC.

En esta baquela se logra una reducción en tamaño del $41 \%$ en comparación con los prototipos anteriores, puesto que se reduce la adquisición de los datos digitales y el procesado. Por medio de un conector se hace el link con la otra tarjeta que es la parte de la potencia en la cual van alojados el driver y los relés.

\section{Etapa de potencia}

Como es conocido a nivel industrial, las señales son a 24 VDC y el microcontrolador maneja señales de $5 \mathrm{~V}$ así que se debe acoplar a $24 \mathrm{~V} \mathrm{DC}$ nuevamente. Para ello se utilizan los relés.

El PIC no es un dispositivo diseñado para corrientes altas, por tanto no puede energizar un relé para hacer que conmute, ya que a pesar de que las salidas del micro son de $5 \mathrm{Vdc}$, al conectarlas directo al relé, debido a la corriente que consume el voltaje se reduce a 1,3 Vdc aproximadamente, lo cual hace que no alcance a energizarse como para conmutar.

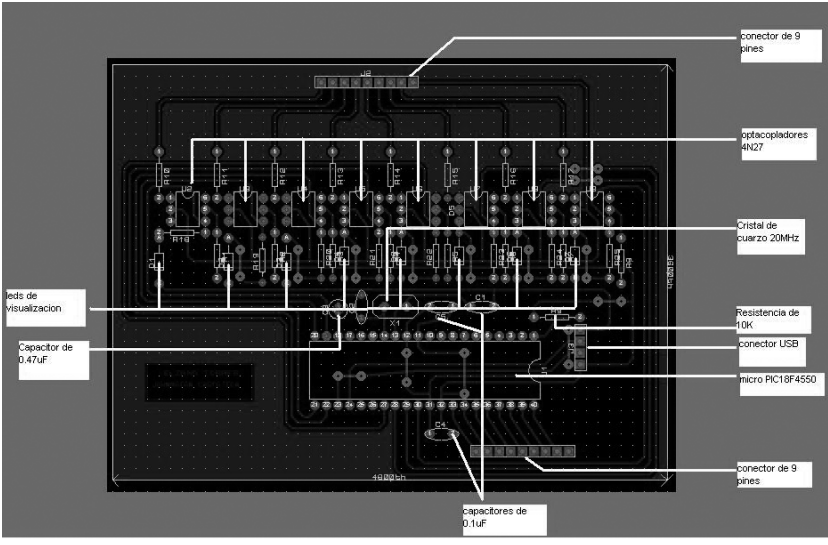

Figura 8. Diagrama final del diseño de la tarjeta. Fuente: los autores

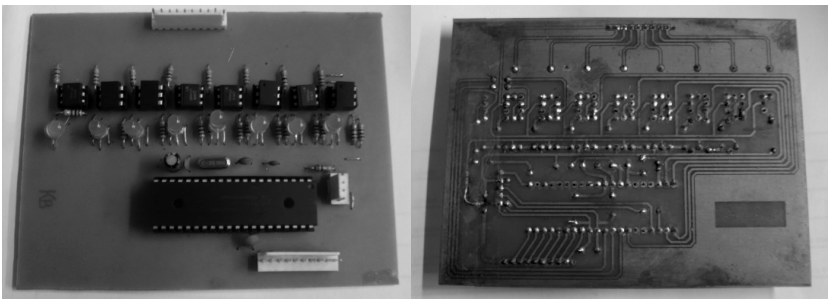

Figura 9. Tarjeta del prototipo final. Fuente: Los autores

Para la solución de este inconveniente se utiliza un dispositivo capaz de soportar corrientes más altas pero sin tener que cambiar el nivel de $5 \mathrm{Vdc}$ con el cual funcionan los relevos; el driver ULN2803 proporciona una corriente de salida de hasta $500 \mathrm{~mA}$ y voltajes en las señales de entrada de hasta 30 Vdc puesto que está diseñado en una configuración tipo Darlington.

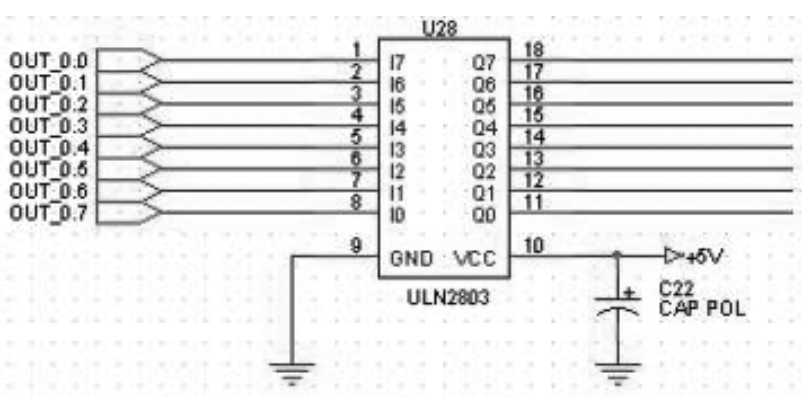

Figura 10. Diagrama de conexión del Driver ULN2803.

Tomado de http://www. datasheetcatalog.net/es/datasheets_pdf/ U/L/N/2/ULN2803-D.shtm

Este dispositivo puede ser alimentado por una fuente diferente a las señales de entrada, lo cual es favorable, porque el puerto USB no proporciona la corriente necesaria para la conmutación de los relés ya que este proporciona una corriente máxima de $200 \mathrm{~mA}$ corriendo el riesgo de dañar el puerto al sobrepasarla en el momento que se necesiten todas las salidas activas. 
Al igual que en la implementación de los opto acopladores, la configuración y funcionamiento es muy importante para su correcta utilización. Uno de los mayores inconvenientes presentados en la implementación del driver consistió en que se tenía la idea que proporcionaba a su salida un uno lógico o un nivel de voltaje alto y la duda de unir la tierra de alimentación con la tierra del microcontrolador ya que en las primeras pruebas del funcionamiento de las salidas estas no funcionaban. Tras una investigación más profunda sobre la utilización y funcionamiento del ULN2803 se logró su implementación y correcto uso dentro del entrenador, como no envía un nivel de voltaje sino una tierra de potencia que es referencia del voltaje de alimentación y la unión de las dos tierras para que el driver "entienda" las señales provenientes del micro controlador.

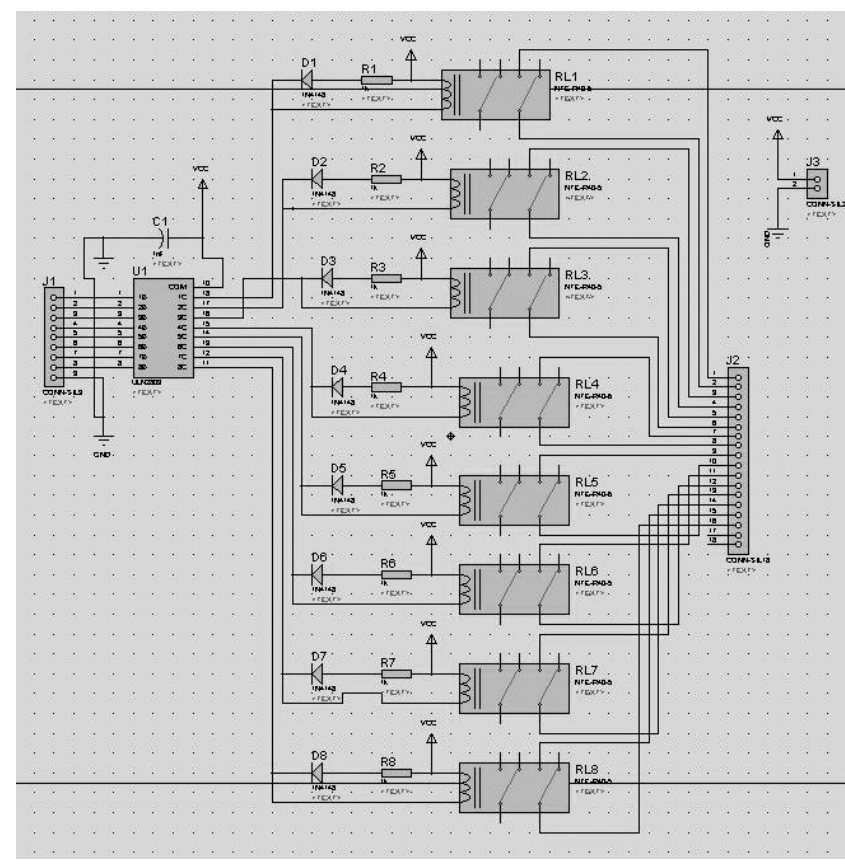

Figura 11. Diagrama de la etapa de potencia del prototipo. Fuente: Los autores

La conexión de los relevadores se determinó a partir del funcionamiento del driver. Conectando un pin de la bobina directo a la salida del driver, el otro a 5 Vdc que es la misma alimentación del ULN 2803 con una resistencia de $1 \mathrm{k}$ para limitar la corriente en serie con un diodo LED para proteger la salida y visualizar su correcto funcionamiento.

El diseño de la tarjeta de potencia se realizó una vez aclaradas las dudas del funcionamiento del ULN 2803 y de las pruebas de energización de los relés, para el diseño se recurrió al Software Proteus en el cual se realiza el circuito electrónico y el impreso de esta etapa del sistema.

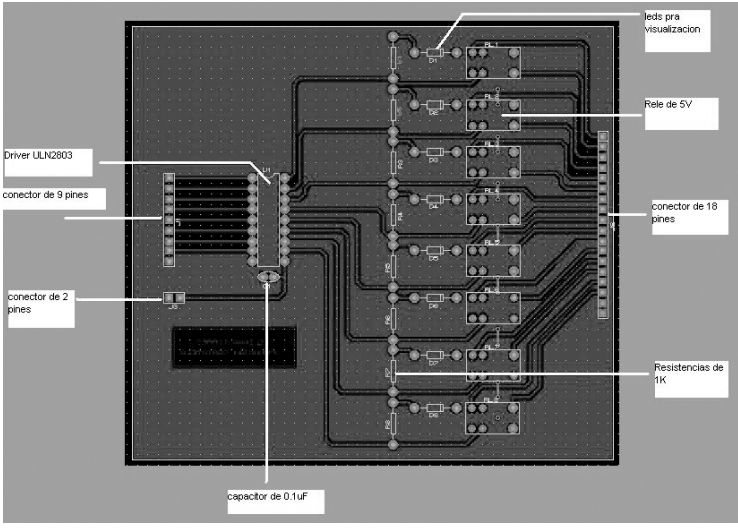

Figura 12. Diseño final de la tarjeta de potencia. Fuente: Los autores

\section{Resultados}

Pruebas del sistema

Las pruebas de comunicación se realizaron en dos etapas:

La primera parte para lograr la comunicación del PIC con el PC requiere de la programación del microcontrolador con un programa en el cual el computador reconozca al PIC como un dispositivo USB (de la misma forma como reconoce las memorias USB), el código fuente en $C$ configura los pines de comunicación D+ y D- del micro controlador e implementa rutinas de interrupción donde el micro controlador envía y recibe datos provenientes del PC.

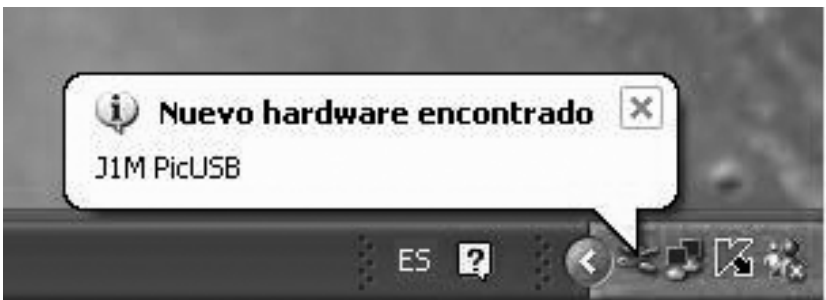

Figura 13. Reconocimiento del dispositivo en el computador Fuente: los autores

En el computador también se desarrolla un programa en $\mathrm{C}$ con el DEV C++ que sirve como interfase entre el estudiante y el microcontrolador. Una vez logrado el reconocimiento del PIC por parte del PC se procede a enviar un dato. El dato a enviar en las primeras pruebas es un número entero. Es enviado al PIC, el cual debe enviar este mismo dato binario al puerto B configurado como salida. Para visualizar esto se utilizan LEDS.

\section{Pruebas con hardware}

Etapa de suma importancia, puesto que es aquí donde se implementa el entrenador como tal. A pesar 
de que para cada etapa se realizaron pruebas por aparte, es decir, de los opto acopladores, del driver y los relevos. Se debe asegurar el funcionamiento pero con las señales provenientes del micro controlador y las provenientes de la etapa de acondicionamiento por parte de los opto acopladores.

En una segunda prueba se realiza un programa sencillo donde se utilicen funciones básicas como AND y OR. El programa se realiza en AWL y las instrucciones son muy similares al software PLC Simulator.

\section{Ejemplo}

Al igual que en las primera prueba se visualizan las salidas a través de LEDS.

\section{Producto final}

\begin{tabular}{|c|c|}
\hline \multicolumn{2}{|l|}{ Función $O R$} \\
\hline 00000000 & Se aseguran las salidas a cero lógico \\
\hline LD 0 & Carga de la entrada 0 \\
\hline OR 1 & Realiza la función OR con la entrada 1 \\
\hline OUT 100 & $\begin{array}{l}\text { Se define la salida } 0 \text {, la cual de activa si cu- } \\
\text { alquiera de las dos entradas esta activa }\end{array}$ \\
\hline END & Fin del programa, teclear enter \\
\hline \multicolumn{2}{|c|}{$\begin{array}{l}\text { Salidal } 00000001 \text { En este caso solo se activa la salida cero al poner } \\
\text { un uno lógico en alguna de las entradas cargadas en el programa. }\end{array}$} \\
\hline \multicolumn{2}{|l|}{ Función AND } \\
\hline 00000000 & Se aseguran las salidas a cero lógico \\
\hline LD 0 & Carga la entrada 0 \\
\hline AND 1 & Realiza la función AND con la entrada 1 \\
\hline OUT 100 & $\begin{array}{l}\text { Define la salida } 0 \text {, la cual de activa si cualquiera } \\
\text { de las dos entradas esta activa }\end{array}$ \\
\hline END & Fin del programa, teclear enter \\
\hline
\end{tabular}

\section{Conclusiones}

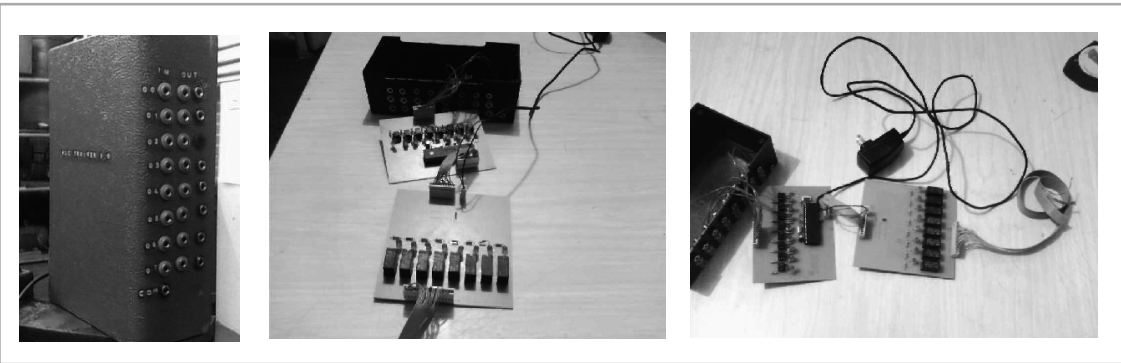

Figura 14. Imágenes del producto final PCL trainer 9.0. Fuente: los autores

- Fue posible el diseño e implementación de un entrenador en lista de instrucciones, basado en la necesidad de la comunidad UNIMINUTO de adquirir competencias en este campo ofreciendo a los estudiantes una novedosa ventaja con el fin de lograr habilidades para poder desempeñarse óptimamente en un cargo tecnológico.

El uso de recursos tecnológicos enfocados al área, facilita el desarrollo de un proyecto; profundiza y aclara conocimientos para la solución de posibles problemas durante su ejecución.

- La investigación ordenada y centralizada es parte fundamental en la solución de problemas y planteamiento y diseño de sistemas o mejoras de estos.

Con la implementación del entrenador en lista de instrucciones, los estudiantes tendrán la posibilidad de aplicar los conocimientos sobre el control automático y su campo de aplicación.

El conocimiento más detallado de los dispositivos a utilizar permite la detección y solución de los inconvenientes presentados durante el diseño y la implementación de un sistema, haciendo necesaria una investigación profunda y la realización de pruebas en cada etapa del sistema.

Configurar un puerto análogo, para la lectura y salida de señales de este tipo, sería una mejora al entrenador; que permitiría el control y visualización de variables físicas en tiempo real.

- Para un correcto funcionamiento del entrenador, es necesario tener en cuenta el limite establecido de corriente en las salidas a relevador y no sobre pasar el nivel de voltaje en las entradas digitales de las tarjetas. 


\section{Referencias}

[1] DatasheetCatalog.com (s.f.). Recuperado el 13 de Febrero de 2009, de http://micros.mforos. $\mathrm{com} / 1$ 149901/6229274-problema-con-comunicacion-pc-pic-por-usb/

[2] DatasheetCatalog.com (s.f.). Recuperado el 13 de Febrero de 2009, de www. datasheetcatalog.net/ es/datasheets_pdf/4/N/2/5/4N25.shtml

[3] Electrónica estudio.com (s.f.). Recuperado el 13 de Febrero de 2009, de http://www.electronicaestudio.com/intermedios.htm\#5i_or
[4] Microchip (s.f.). Recuperado el 13 de Febrero de 2009, de www.microchip.com/stellent/ idcplg?ldcService $=$ SS_GET_PAGE\&nodeld $=1335 \& d D$ ocName $=$ en010300

[5] Micros.mforos.com (s.f.). Recuperado el 13 de Febrero de 2009, de http://www. datasheetcatalog.net/ es/datasheets_pdf/U/L/N/2/ULN2803-D.shtml

[6] Scribd (s.f.). Recuperado el 13 de Febrero de 2009, de www.scribd.com/doc/2940003/PIC18F4550

[7] uControl (s.f.). Recuperado el 13 de Febrero de 2009, de http://www.ucontrol.com.ar/wiki/index. php?title=modulo_8_E/S

John William Vasquez C. Ingeniero Electrónico, Universidad del Valle, Calli, Colombia. M.Sc. en Automatización e Informática, Universidad Politécnica de Valencia, España. Coordinador área de Automatización y control, programa de Tecnología en Electrónica, Corporación Universitaria Minuto de Dios (UNIMINUTO), jvasquez@uniminuto.edu

Eliana Torres Niño estudiante de VI semestre de Tecnología en Electrónica, Corporación Universitaria Minuto de Dios (UNIMINUTO). emator151@hotmail.com

Johnnatan Castañeda estudiante de VI semestre de Tecnología en Electrónica, Corporación Universitaria Minuto de Dios (UNIMINUTO), johnnatan_cas@hotmail.com 